\title{
Vertical and complete lifts from a manifold to its cotangent bundle
}

\author{
By K. YANO and E. M. PATterson
}

(Received Aug. 10, 1966)

\section{$\S 1$. Introduction.}

Let $M$ be a differentiable manifold of class $C^{\infty}$ and dimension $n$, and let ${ }^{c} T(M)$ be the cotangent bundle of $M$ : that is, the bundle of covariant vectors in $M$. Then ${ }^{c} T(M)$ is also a differentiable manifold of class $C^{\infty}$; the dimension of ${ }^{c} T(M)$ is $2 n$.

In this paper we consider methods by which certain types of tensor fields in $M$ can be extended to ${ }^{c} T(M)$ so as to give useful information about the relationships between the structures of the two manifolds. We call extensions of this kind lifts of the tensor fields in $M$ and consider two main types of lifts, which we call vertical lifts and complete lifts respectively. Our main interest focuses on complete lifts of vector fields, tensor fields of type $(1,1)$ and skew-symmetric tensor fields of type $(1,2)$. In each of these cases we define the complete lift to be a tensor field of the same type as the original. In general, the vertical lift of a tensor field does not have the same type as the original; nevertheless the construction is a useful one.

Our methods enable us to examine the structure of ${ }^{c} T(M)$ in relation to that of $M$. In particular, we show how almost complex and similar structures on $M$ can be extended to ${ }^{c} T(M)$. We also examine lifts of affine connections in $M$, using the idea of a Riemann extension ([4], [5], [6]).

The methods used and the results obtained are to some extent similar to results previously established for tensor fields in the tangent bundle of a differentiable manifold ([1], [3], [7], [8], [9], [12], [13], [14], [15]). However there are various important differences and it appears that the problem of extending tensor fields to the cotangent bundle presents difficulties which are not encountered in the case of the tangent bundle.

Throughout we use the following notations and conventions:

1. $\pi:{ }^{c} T(M) \rightarrow M$ is the projection of ${ }^{c} T(M)$ onto $M$.

2. Suffixes $A, B, C, D$ take the values 1 to $2 n$. Suffixes $a, b, c, \cdots, h, i, j, \cdots$ take the values 1 to $n$ and $i=i+n$, etc.. The summation convention for repeated indices is used. Whenever notations such as $\left(\varepsilon_{C B}\right),\left(\varepsilon^{B A}\right),\left(F_{B}^{A}\right)$ are used 
for matrices, the suffix on the left indicates the column and the suffix on the right indicates the row.

3. $\mathscr{T}_{s}^{r}(M)$ denotes the set of tensor fields of class $C^{\infty}$ and type $(r, s)$ in $M$. Similarly $\mathscr{T}_{s}^{r}\left({ }^{c} T(M)\right)$ denotes the corresponding set of tensor fields in ${ }^{c} T(M)$.

4. Vector fields in $M$ are denoted by $X, Y, Z$. The Lie product of $X$ and $Y$ is denoted by $[X, Y]$. The Lie derivative with respect to $X$ is denoted by $\mathcal{L}_{X}$. Tensor fields of type $(1,1)$ are denoted by $F, G$ and tensor fields of type $(1,2)$ by $S, T$.

\section{$\S 2$. The basic 1-form in ${ }^{c} T(M)$.}

If $A$ is a point in $M$, then $\pi^{-1}(A)$ is the fibre over $A$. Any point $P \in \pi^{-1}(A)$ is an ordered pair $\left(A, p_{A}\right)$, where $p$ is a 1 -form in $M$ and $p_{A}$ is its value at $A$. Suppose that $U$ is a coordinate neighbourhood in $M$ such that $A \in U$. Then $U$ induces a coordinate neighbourhood $\pi^{-1}(U)$ in ${ }^{c} T(M)$ and $P \in \pi^{-1}(U)$. If $A$ has coordinates $\left(x^{1}, x^{2}, \cdots, x^{n}\right)$ relative to $U$ and $p_{A}$ has components $\left(p_{1}, p_{2}, \cdots, p_{n}\right)$, then $P$ has coordinates $\left(x^{1}, x^{2}, \cdots, x^{n}, p_{1}, p_{2}, \cdots, p_{n}\right)$ relative to $\pi^{-1}(U)$. If $U^{*}$ is another coordinate neighbourhood in $M$ containing $A$, then $\pi^{-1}\left(U^{*}\right)$ contains $P$ and the coordinates of $P$ relative to $\pi^{-1}\left(U^{*}\right)$ are $\left(x^{* 1}, x^{* 2}\right.$, $\left.\cdots, x^{* n}, p_{1}^{*}, p_{2}^{*}, \cdots, p_{n}^{*}\right)$ where

$$
p_{i}^{*}=p_{j} \frac{\partial x^{j}}{\partial x^{* i}},
$$

the derivatives being evaluated at $A$.

Let $p$ be the 1 -form in ${ }^{c} T(M)$ whose components relative to $\pi^{-1}(U)$ are $\left(p_{1}, \cdots, p_{n}, 0, \cdots, 0\right)$. By $(2.1)$, the components of $p$ relative to $\pi^{-1}\left(U^{*}\right)$ are $\left(p_{1}^{*}, \cdots, p_{n}^{*}, 0, \cdots, 0\right)$. In fact we can write

$$
p=p_{i} d x^{i}=p_{i}^{*} d x^{* i} \text {. }
$$

We call $p$ the basic 1-form in ${ }^{c} T(M)$.

The exterior derivative $d p$ of $p$ is the 2 -form given by

$$
d p=d p_{i} \wedge d x^{i}
$$

in $\pi^{-1}(U)$. Hence, if $d p=\frac{1}{2} \varepsilon_{C B} d x^{C} \wedge d x^{B}$, (where $d x^{\bar{i}}=d p_{i}$ ), we have

$$
\left(\varepsilon_{C B}\right)=\left(\begin{array}{rr}
0 & I \\
-I & 0
\end{array}\right)
$$

where $I$ is the unit $n \times n$ matrix.

Since the matrix $\left(\varepsilon_{C B}\right)$ in $(2.2)$ is non-singular, it has an inverse. Denoting this by $\left(\varepsilon^{B A}\right)$, so that

$$
\varepsilon_{C B} \varepsilon^{B A}=\delta_{C}^{A},
$$


we have

$$
\left(\varepsilon^{B A}\right)=\left(\begin{array}{rr}
0 & -I \\
I & 0
\end{array}\right)
$$

We shall write $\varepsilon^{-1}$ for the tensor field of type $(2,0)$ whose components in $\pi^{-1}(U)$ are $\varepsilon^{B A}$. This tensor field is of importance in our construction of complete lifts.

\section{$\S 3$. The vertical lift of a function.}

If $f$ is a function in $M$, we write $f^{v}$ for the function in ${ }^{c} T(M)$ obtained by forming the composition of $\pi$ and $f$, so that

Thus if $(A, p) \in \pi^{-1}(U)$, then

$$
f^{v}=f \circ \pi \text {. }
$$

$$
f^{v}(A, p)=(f \circ \pi)(A, p)=f(A) .
$$

Thus the value of $f^{v}$ is constant along each fibre, being equal to the value of $f$ at the point on the fibre in the base space. We call $f^{V}$ the vertical lift of the function $f$.

\section{$\S 4$. The vertical lift of a vector field.}

If $X \in I_{0}^{1}(M)$ (so that $X$ is a vector field in $M$ ) we write $X^{v}$ for the function in ${ }^{c} T(M)$ defined by

$$
X^{v}(A, p)=p\left(X_{A}\right)
$$

where $X_{A}$ is the vector obtained by evaluating $X$ at $A$. Thus if $X^{h}$ are the components of $X$ in $U$ at the point $A$, then $X^{v}$ is the mapping $(A, p) \rightarrow p_{i} X^{i}$.

We call $X^{v}$ the vertical lift of the vector field $X$. We have $X^{v} \in \mathscr{I}_{0}^{0}\left({ }^{c} T(M)\right)$, since $X^{v}$ is by definition a function in ${ }^{c} T(M)$.

We observe that if $P \in M$, then $X^{v}(P)=0$.

\section{$\S 5$. The determination of vector fields in ${ }^{c} T(M)$.}

Suppose that $\tilde{X} \in \mathscr{I}_{0}^{1}\left({ }^{c} T(M)\right)$. Then $\tilde{X}$ is completely determined by its action on functions of class $C^{\infty}$ in ${ }^{c} T(M)$. In $\S 4$ we introduced a special type of function in ${ }^{c} T(M)$, namely the vertical lift of a vector field in $M$. We now show that any element $\tilde{X}$ of $\mathscr{I}_{0}^{1}\left({ }^{c} T(M)\right)$ is completely determined by its action on functions of this type.

Proposition 1. Let $\tilde{X}$ and $\tilde{Y}$ be vector fields in ${ }^{c} T(M)$ such that

$$
\tilde{X} Z^{v}=\tilde{Y} Z^{v}
$$

for all $Z \in \mathscr{I}_{0}^{1}(M)$. Then $\tilde{X}=\tilde{Y}$. 
Proof. It is sufficient to show that if $\tilde{X} Z^{v}=0$ for all $Z \in \mathscr{I}_{0}^{1}(M)$, then $\tilde{X}$ is zero.

If $Z$ is the vector field with components $Z^{n}$ in $U$, then

$$
\tilde{X} Z^{v}=\tilde{X}^{i} \partial_{i}\left(p_{a} Z^{a}\right)+\tilde{X}^{\bar{i}} \partial_{\bar{i}}\left(p_{a} Z^{a}\right),
$$

where $\tilde{X}^{A}$ are the components of $\tilde{X}$. Hence, if $\tilde{X} Z^{v}=0$ for all $Z \in \mathscr{I}_{0}^{1}(M)$, we have

for all $Z$.

$$
p_{a} \tilde{X}^{i} \partial_{i} Z^{a}+\tilde{X}^{i} Z^{i}=0
$$

Choose $Z$ to be the vector field given in $U$ by $Z^{i}=\delta_{j}^{i}$. Then from (5.1) we get

$$
\tilde{X}^{\bar{j}}=0
$$

Hence (5.1) becomes

$$
p_{a} \tilde{X}^{i} \partial_{i} Z^{a}=0
$$

for all $Z$.

Let $i, j$ be fixed integers such that $1 \leqq i \leqq n$ and $1 \leqq j \leqq n$. Choose $Z$ to be the vector field given in $U$ by

$$
Z^{j}=x^{i}, Z^{a}=0(a \neq j) .
$$

Then from (5.3) we get

$$
p_{j} \tilde{X}^{i}=0
$$

It follows that we have

$$
\tilde{X}^{i}=0
$$

at all points of ${ }^{c} T(M)$ except possibly those at which all the components $p_{1}, \cdots, p_{n}$ are zero: that is, at points of the base space. However, the components of $\tilde{X}$ are continuous (since they are of class $C^{\infty}$ ) and so $\tilde{X}^{i}$ is also zero at points of the base space.

Hence $\tilde{X}^{i}=0$ for all points of $\pi^{-1}(U)$. This holds for each $i$ satisfying $1 \leqq i \leqq n$. Therefore, using (5.2), $\tilde{X}$ is the zero vector in $\pi^{-1}(U)$. From this it quickly follows that $\tilde{X}=0$ in ${ }^{c} T(M)$.

\section{$\S 6$. Vertical vectors.}

Let $\left.\tilde{X} \in \mathscr{I}_{0}^{1}{ }^{c} T(M)\right)$ be such that $\tilde{X} f^{v}=0$ for all $f \in \mathscr{I}_{0}^{0}(M)$. Then we say that $\tilde{X}$ is a vertical vector field. It is easily shown that $\tilde{X}$ is vertical if and only if its components in $\pi^{-1}(U)$ satisfy

$$
\tilde{X}^{i}=0 \quad(i=1,2, \cdots, n) .
$$

In $\S 7$ and $\S 8$ we introduce two types of vertical vector fields in ${ }^{c} T(M)$, constructed respectively from 1 -forms and from tensor fields of type $(1,1)$ in $M$. 


\section{$\S 7$. The vertical lift of a 1 -form.}

Suppose that $\omega \in \mathscr{I}_{1}^{0}(M)$, so that $\omega$ is a 1 -form in $M$. Let $A$ be a point of $M$ and let $U, U^{*}$ be coordinate neighbourhoods containing $A$. If $\omega$ has components $\omega_{i}$ and $\omega_{i}^{*}$ relative to $U$ and $U^{*}$ respectively, then

$$
\omega_{i}^{*}=\omega_{j} \frac{\partial x^{j}}{\partial x^{* i}},
$$

where the derivatives are evaluated at $A$.

Equations (2.1) and (7.1) show that the vector which has components $\left(0, \cdots, 0, \omega_{1}, \cdots, \omega_{n}\right)$ relative to $\pi^{-1}(U)$ at a point $(A, p)$ on the fibre over $A$ has components $\left(0, \cdots, 0, \omega_{1}^{*}, \cdots, \omega_{n}^{*}\right)$ relative to $\pi^{-1}\left(U^{*}\right)$. We call the vector field determined by the vectors which have these components the vertical lift $\omega^{V}$ of $\omega$. Thus $\omega^{V} \in \mathscr{I}_{0}^{1}\left({ }^{c} T(M)\right)$.

Clearly

$$
\omega^{V}\left(f^{V}\right)=0
$$

so that $\omega^{V}$ is a vertical vector.

By Proposition 1, $\omega^{V}$ is completely determined by its action on functions in ${ }^{c} T(M)$ of the form $Z^{v}$. Since

we have

$$
\omega_{i} \frac{\partial}{\partial p_{i}}\left(p_{j} Z^{j}\right)=\omega_{j} Z^{j}
$$

$$
\omega^{v}\left(Z^{v}\right)=\{\omega(Z)\}^{v}
$$

If $\omega, \tau \in \mathscr{T}_{1}^{0}(M)$ and $f \in \mathscr{I}_{0}^{0}(M)$, it is easily proved that

$$
\begin{gathered}
(\omega+\tau)^{V}=\omega^{V}+\tau^{V}, \\
(f \omega)^{V}=f^{V} \omega^{V} .
\end{gathered}
$$

\section{$\S 8$. The vertical lift of a tensor field of type $(1,1)$.}

Suppose now that $F \in \mathscr{I}_{1}^{1}(M)$. If $F$ has components $F_{i}^{h}$ and $F_{i}^{* h}$ relative to $U$ and $U^{*}$ respectively, then

$$
p_{a}^{*} F_{i}^{* a}=p_{b} F_{j}^{b} \frac{\partial x^{j}}{\partial x^{* i}} .
$$

Hence, using (2.1) and (8.1), the vector which has components $\left(0, \cdots, 0, p_{a} F_{1}{ }^{a}\right.$, $\left.\cdots, p_{a} F_{n}{ }^{a}\right)$ relative to $\pi^{-1}(U)$ has components $\left(0, \cdots, 0, p_{a}^{*} F_{1}^{* a}, \cdots, p_{a}^{*} F_{n}^{* a}\right)$ relative to $\pi^{-1}\left(U^{*}\right)$. We call the vector field determined by the vectors which have these components the vertical lift $F^{v}$ of $F$.

The vector field $F^{v}$ is unlike the vertical lift $\omega^{V}$ of a 1 -form in that the components of $F^{V}$ are not the same at all points of the same fibre. In fact 
$F^{v}$ is zero at points on the base space $M$. Clearly

$$
F^{v}\left(f^{v}\right)=0
$$

so that $F^{v}$ is vertical.

By Proposition 1, $F^{v}$ is completely determined by its action on functions in ${ }^{c} T(M)$ of the form $Z^{v}$. We have

$$
F^{v}\left(Z^{v}\right)=(F(Z))^{v}
$$

If $F, G \in \mathscr{I}_{1}^{1}(M)$, then

$$
(F+G)^{v}=F^{v}+G^{v}
$$

and if $f \in \mathscr{I}_{0}^{0}(M)$, then

$$
(f F)^{v}=f^{v} F^{v} .
$$

\section{$\S 9$. The complete lift of a vector field.}

In $\S 7$ and $\S 8$ we constructed vector fields in ${ }^{c} T(M)$. from 1 -forms and tensors of type $(1,1)$, in $M$. Constructions such as these can be carried out for other types of tensor field in $M$, but they have the disadvantage of changing the type of the tensor fields under consideration in going from $M$ to ${ }^{c} T(M)$. Thus there seems to be no obvious way in which such a construction lifts a vector field in $M$ to a vector field in ${ }^{c} T(M)$. However, we now describe a different process by which we can lift vector fields. Subsequently we shall apply similar methods to tensor fields of type $(1,1)$ and skew-symmetric tensor fields of type $(1,2)$, in each case obtaining tensor fields of the same type. In our construction we use the tensor $\varepsilon^{-1}$ introduced in $\S 2$.

Suppose that $X \in I_{0}^{1}(M)$. Let $A$ be a point of $M$ and let $U$ be a coordinate neighbourhood containing $A$. We have already defined the vertical lift $X^{v}$ of $X$ to be a function in ${ }^{c} T(M)$. The exterior derivative $d X^{v}$ is the 1 form in ${ }^{c} T(M)$ given in $\pi^{-1}(U)$ by

$$
d X^{V}=p_{a} \frac{\partial X^{a}}{\partial x^{b}} d x^{b}+X^{a} d p_{a} .
$$

We define a vector field $X^{c}$ in ${ }^{c} T(M)$ by $X^{c}=\left(d X^{V}\right) \varepsilon^{-1}$. In $\pi^{-1}(U)$, the components of $X^{c}$ are

$$
\left(X^{1}, X^{2}, \cdots, X^{n},-p_{a} \frac{\partial X^{a}}{\partial x^{1}}, \cdots,-p_{a} \frac{\partial X^{a}}{\partial x^{n}}\right) .
$$

We call $X^{0}$ the complete lift of the vector field $X$. We have

$$
X^{c} f^{V}=(X f)^{V}
$$

and

$$
X^{c} Z^{v}=[X, Z]^{v} .
$$


By Proposition 1, $X^{C}$ is completely determined by (9.2), If $X$ and $Y \in \mathfrak{I}_{0}^{1}(M)$, then

$$
(X+Y)^{C}=X^{C}+Y^{C} .
$$

\section{$\S 10$. Projectable vectors.}

The vector field $X^{c}$ is completely determined by its first $n$ components and in particular $X^{c}$ is zero if these components are zero. An alternative way of expressing this is to say that $X^{C}$ is zero if it is vertical.

If $\tilde{X} \in \mathscr{I}_{0}^{1}\left({ }^{c} T(M)\right)$ and if there exists $X \in \mathscr{I}_{0}^{1}(M)$ such that $\tilde{X}-X^{c}$ is vertical then we shall say that $\tilde{X}$ is projectable, with projection $X$. A necessary and sufficient condition for $\tilde{X}$ to be projectable with projection $X$ is that the components $\tilde{X}^{A}$ of $\tilde{X}$ at a point $(A, p)$ in $\pi^{-1}(U)$ are related to the components $X^{h}$ of $X$ at $A$ by

$$
\tilde{X}^{h}=X^{h} \quad(h=1, \cdots, n) .
$$

Thus the components $\tilde{X}^{h}$ are constant along any fibre.

We observe that the complete lift $X^{c}$ of any $X \in \mathscr{I}_{0}^{1}(M)$ is projectable with projection $X$, for $X^{c}-X^{c}$ is trivially vertical.

\section{$\S 11$. The tangent space of ${ }^{c} T(M)$.}

If $\Phi$ denotes the algebra of functions of class $C^{\infty}$ in ${ }^{c} T(M)$ and $\mathscr{X}$ denotes the $\Phi$-module of vector fields in ${ }^{c} T(M)$, then a tensor field in ${ }^{c} T(M)$ of type $(0, r)$ (respectively $(1, r)$ ), where $r$ is a positive integer, can be regarded as an $r$-linear mapping of $\mathfrak{X}^{r}$ into $\Phi$ (respectively $\mathscr{X}$ ), where $\mathfrak{X}^{r}$ is the Cartesian product of $r$ copies of $\mathscr{X}$. (See [2], p. 26.)

The following result, which should be compared with Proposition 1, is used frequently in the sequel.

Proposition 2. Let $\hat{S}, \tilde{T}$ be tensor fields in ${ }^{c} T(M)$ of type $(0, r)$ or $(1, r)$ such that

$$
\tilde{S}\left(\tilde{X}_{(1)}, \cdots, \tilde{X}_{(r)}\right)=\tilde{T}\left(\tilde{X}_{(1)}, \cdots, \tilde{X}_{(r)}\right)
$$

for all vector fields $\tilde{X}_{(s)}(s=1, \cdots, r)$ which are complete lifts of vector fields in M. Then

$$
\widetilde{S}=\widetilde{T} \text {. }
$$

Proof. We shall consider the case of tensor fields of type $(1,2)$. It is easily seen that the argument extends without difficulty to the other cases. Moreover (in the general case) it is sufficient to show that if

$$
\widetilde{S}\left(\tilde{X}_{(1)}, \cdots, \tilde{X}_{(r)}\right)=0
$$

for all vector fields $\tilde{X}_{(s)}(s=1, \cdots, r)$ which are complete lifts of vector fields 
in $M$, then $\widehat{S}=0$.

Let $U$ be a coordinate neighbourhood in $M$ and let $\pi^{-1}(U)$ be the induced neighbourhood in ${ }^{c} T(M)$. Let $\hat{S} \in \mathscr{I}_{2}^{1}\left({ }^{c} T(M)\right)$ be such that

$$
\widetilde{S}\left(X^{c}, Y^{c}\right)=0
$$

for all $X, Y \in \mathscr{I}_{0}^{1}(M)$. Suppose that $X, Y$ have components $X^{n}, Y^{h}$ respectively in $U$. Then the components of $\hat{S}$ satisfy

$$
\begin{gathered}
\hat{S}_{i \hbar}{ }^{A} X^{i} Y^{h}-\hat{S}_{\bar{i}}{ }^{A}\left(p_{a} \partial_{i} X^{a}\right) Y^{h}-\widetilde{S}_{i \bar{h}}{ }^{A} X^{i}\left(p_{a} \partial_{h} Y^{a}\right) \\
+\hat{S}_{\bar{i} \bar{h}^{A}}{ }^{A}\left(p_{a} \partial_{i} X^{a}\right)\left(p_{b} \partial_{h} Y^{b}\right)=0 .
\end{gathered}
$$

Choose $X, Y$ to be the vector fields given in $U$ by $X^{i}=\delta_{k}^{i}$ and $Y^{h}=\delta_{j}^{n}$. Then from (11.1) we get

$$
\widehat{S}_{k j}^{A}=0
$$

Next choose $X, Y$ to be given by

$$
X^{i}=\delta_{b}^{i} x^{k}, \quad Y^{h}=\delta_{j}^{h},
$$

where $b, k$ are fixed. Then, from [11.1) and (11.2) we get

Hence

$$
\hat{S}_{\bar{k} j}{ }^{A} p_{b}=0 \text {. }
$$

$$
\hat{\mathrm{S}}_{\bar{k}_{j}}{ }^{A}=0
$$

at all points of ${ }^{c} T(M)$ except possibly those at which all the components $p_{1}, \cdots, p_{n}$ are zero: that is, at points of the base space. However the components of $\hat{S}$ are continuous; hence we have equations (11.3)] at all points of ${ }^{c} T(M)$.

Similarly we can show that

$$
\widetilde{S}_{k \bar{j}}{ }^{A}=0 .
$$

Finally, by choosing $X, Y$ to be given by

$$
X^{i}=\delta_{b}^{i} x^{k}, \quad Y^{h}=\delta_{a}^{h} x^{j}
$$

and using (11.1) in conjunction with (11.2), (11.3) and (11.4), we can show by a similar argument that

$$
\widetilde{S}_{\bar{k} \bar{j}}^{A}=0 \text {. }
$$

From (11.2), (11.3), (11.4) and (11.5) it follows that $\hat{S}$ is zero in $\pi^{-1}(U)$. Hence $\hat{S}$ is the zero tensor field.

\section{$\S 12$. The vertical lift of a tensor field of type $(1,2)$.}

Suppose that $S \in \mathscr{I}_{2}^{1}(M)$ and that $S$ has components $S_{j i}{ }^{h}$ at a point $A$ in a coordinate neigbourhood $U$. At the point $(A, p)$ in $\pi^{-1}(U)$, we can define a 
tensor $\tilde{P}$ of type $(0,2)$ with components given by

$$
\begin{aligned}
& \tilde{P}_{j i}=p_{a} S_{j i}{ }^{a}, \tilde{P}_{\overline{j i}}=0, \\
& \tilde{P}_{j \bar{i}}=0, \quad \tilde{P}_{\bar{j} \bar{i}}=0 .
\end{aligned}
$$

The tensor $\varepsilon^{-1}$ introduced in $\S 2$ is of type $(2,0)$; hence we can define a tensor of type $(1,1)$ by transvecting with $\varepsilon^{-1}$. We write $S^{V}$ for the tensor field whose components $\widetilde{S}_{B}{ }^{A}$ in $\pi^{-1}(U)$ are given by

Thus

$$
\hat{S}_{B}{ }^{A}=\widetilde{P}_{B C} \varepsilon^{C A} \text {. }
$$

$$
\left(\hat{S}_{B}{ }^{A}\right)=\left(\begin{array}{ll}
0 & 0 \\
Q & 0
\end{array}\right)
$$

where $Q$ is the matrix $\left(p_{a} S_{j i}{ }^{a}\right)$.

We call $S^{V}$ the vertical lift of the tensor field $S$. If $\omega \in \mathscr{I}_{1}^{0}(M)$, then

and if $Z \in \mathscr{I}_{0}^{1}(M)$, then

$$
S^{v}\left(\omega^{v}\right)=0
$$

$$
S^{v}\left(Z^{C}\right)=\left(S_{Z}\right)^{v}
$$

where $S_{Z}$ is the tensor field of type $(1,1)$ in $M$ defined by

$$
S_{Z}(X)=S(Z, X) \text {. }
$$

By Proposition 2, $S^{v}$ is completely determined by (12.3), Since any vertical vector at any point is linearly dependent on vectors of the form $\omega^{V}$, it follows from (12.2) that

$$
S^{v}(\tilde{Y})=0
$$

for all vertical vector fields $\tilde{Y}$.

\section{$\S 13$. Identities involving vertical and complete lifts.}

In this section we establish various identities concerning vertical and complete lifts, particularly involving Lie products. These are required for subsequent calculations.

Proposition 3. If $\tilde{X}, \tilde{Y}$ are vertical vectors in ${ }^{c} T(M)$, then their Lie product $[\tilde{X}, \tilde{Y}]$ is also vertical.

PROOF. If $f \in I_{0}^{0}(M)$, then

$$
\tilde{X} f^{v}=0=\tilde{Y} f^{v} .
$$

Hence $\quad[\tilde{X}, \tilde{Y}] f^{v}=\tilde{X}\left(\tilde{Y}\left(f^{v}\right)\right)-\tilde{Y}\left(\tilde{X}\left(f^{v}\right)\right)=0$.

Proposition 4. If $\phi, \omega \in \mathscr{T}_{1}^{\circ}(M)$, then

$$
\left[\phi^{v}, \omega^{v}\right]=0 .
$$

Proof. If $Z \in \mathscr{I}_{0}^{1}(M)$, then 


$$
\begin{aligned}
{\left[\phi^{V}, \omega^{V}\right] Z^{V} } & =\phi^{V}\left(\omega^{V}\left(Z^{V}\right)\right)-\omega^{V}\left(\phi^{V}\left(Z^{V}\right)\right) \\
& =\phi^{V}(\omega(Z))^{V}-\omega^{V}(\phi(Z))^{V}
\end{aligned}
$$

by (7.3). Since $\omega(Z), \phi(Z) \in I_{0}^{0}(M)$ and $\phi^{v}, \omega^{V}$ are vertical, we get

$$
\left[\psi^{V}, \omega^{V}\right] Z^{V}=0 \text {. }
$$

Hence, by Proposition 1, $\quad\left[\psi^{v}, \omega^{V}\right]=0$.

Proposinion 5. If $\omega \in \mathscr{T}_{1}^{0}(M)$ and $F \in \mathscr{I}_{1}^{1}(M)$, then

$$
\left[\omega^{v}, F^{v}\right]=\{\omega F\}^{v}
$$

where $\omega F$ is the 1-form defined by $(\omega F)(X)=\omega(F X)$.

Proof. If $Z \in I_{0}^{1}(M)$, then

$$
\begin{aligned}
{\left[\omega^{v}, F^{v}\right] Z^{v} } & =\omega^{v}\left(F^{v}\left(Z^{v}\right)\right)-F^{v}\left(\omega^{v}\left(Z^{v}\right)\right) \\
& =\omega^{v}(F(Z))^{v}=\{\omega(F(Z))\}^{v}
\end{aligned}
$$

by (8.3), (7.3) and (8.2). But also

$$
\{\omega F\}^{v} Z^{v}=\{(\omega F) Z\}^{v}=\{\omega(F(Z))\}^{v}
$$

so that the actions of $\left[\omega^{V}, F^{v}\right]$ and $\{\omega F\}^{v}$ on $Z^{v}$ coincide. Thus, from Proposition 1 , we have

$$
\left[\omega^{v}, F^{v}\right]=\{\omega F\}^{V} .
$$

Proposition 6. If $F, G \in I_{1}^{1}(M)$, then

$$
\left[F^{v}, G^{v}\right]=(F G-G F)^{V} .
$$

Proof. If $Z \in \mathscr{I}_{0}^{1}(M)$, then, by (8.3) and (8.4),

$$
\begin{aligned}
{\left[F^{v}, G^{v}\right] Z^{v} } & =F^{v}\left(G^{v}\left(Z^{v}\right)\right)-G^{v}\left(F^{v}\left(Z^{v}\right)\right) \\
& =F^{v}(G(Z))^{v}-G^{v}(F(Z))^{v} \\
& =\{F(G(Z))-G(F(Z))\}^{v} \\
& =(F G-G F)^{v} Z^{v} .
\end{aligned}
$$

The required result now follows from Proposition 1.

Proposition 7. If $\omega=I_{1}^{0}(M)$ and $X \in I_{0}^{1}(M)$, then

$$
\left[X^{C}, \omega^{V}\right]=\left(\mathcal{L}_{X} \omega\right)^{V} \text {. }
$$

Proof. If $Z \in \mathscr{T}_{0}^{1}(M)$, then, by (7.3), (9.2), (7.2) and (9.1)

$$
\begin{aligned}
{\left[X^{c}, \omega^{v}\right] Z^{v} } & =X^{c}\left(\omega^{V}\left(Z^{v}\right)\right)-\omega^{V}\left(X^{c}\left(Z^{v}\right)\right) \\
& =X^{c}(\omega(Z))^{V}-\omega^{V}[X, Z]^{V} \\
& =(X(\omega(Z)))^{V}-(\omega([X, Z]))^{V} \\
& =\left\{\left(\mathcal{L}_{X} \omega\right)(Z)\right\}^{v}
\end{aligned}
$$


(see [2], p. 32). Hence

$$
\left[X^{c}, \omega^{V}\right] Z^{v}=\left(\mathcal{L}_{X} \omega\right)^{v} Z^{v}
$$

so that, by Proposition 1, $\left[X^{C}, \omega^{V}\right]=\left(\mathcal{L}_{X} \omega\right)^{V}$.

Proposition 8. If $X \in \mathscr{I}_{0}^{1}(M)$ and $F \in \mathscr{I}_{1}^{1}(M)$, then

$$
\left[X^{c}, F^{V}\right]=\left(\mathcal{L}_{X} F\right)^{V} \text {. }
$$

Proof. If $Z \in I_{0}^{1}(M)$, then

$$
\begin{aligned}
{\left[X^{c}, F^{v}\right] Z^{v} } & =X^{c}(F(Z))^{v}-F^{v}[X, Z]^{v} \\
& =[X, F(Z)]^{v}-\{F[X, Z]\}^{v} \\
& =\left(\left(\mathcal{L}_{X} F\right) Z\right)^{v} \\
& =\left(\mathcal{L}_{X} F\right)^{v} Z^{v}
\end{aligned}
$$

(see [2], p. 32).

Proposition 9. If $X, Y \in I_{0}^{1}(M)$, then

$$
\left[X^{C}, Y^{C}\right]=[X, Y]^{C} \text {. }
$$

ProOF. If $Z \in \mathscr{I}_{0}^{1}(M)$, then, by (9.2),

$$
\begin{aligned}
& {\left[X^{c}, Y^{c}\right] Z^{v}=X^{c}[Y, Z]^{v}-Y^{c}[X, Z]^{v}} \\
& =[X,[Y, Z]]^{V}-[Y,[X, Z]]^{V} \\
& =[[X, Y], Z]^{V}
\end{aligned}
$$

by the Jacobi identity. Hence

$$
\left[X^{C}, Y^{C}\right] Z^{v}=[X, Y]^{c} Z^{v} \text {. }
$$

Proposition 10. If $S, T \in \mathscr{I}_{2}^{1}(M)$ and $F \in \mathscr{I}_{1}^{1}(M)$, then

$$
\begin{aligned}
& S^{v} T^{v}=0 \\
& S^{v} F^{v}=0 .
\end{aligned}
$$

Proof. By definition, $S^{v}, T^{v} \in \mathcal{I}_{1}^{1}\left({ }^{c} T(M)\right)$. Hence $S^{v} T^{v}$ is also a tensor of type $(1,1)$. If $Z \in \mathscr{I}_{0}^{1}(M)$, then, by (12.3) and (12.4),

$$
S^{v} T^{v}\left(Z^{c}\right)=S^{v}\left(T^{v}\left(Z^{c}\right)\right)=S^{v}\left(T_{z}\right)^{v}=0 .
$$

Hence, by Proposition 2, $S^{v} T^{v}=0$.

Also $F^{V}$ is a vertical vector field in ${ }^{c} T(M)$ and so, by $(12.4), S^{v} F^{v}=0$.

\section{$\S 14$. The complete lift of a tensor field of type $(1,1)$.}

Suppose now that $F \in \mathscr{I}_{1}^{1}(M)$ and that $F$ has components $F_{i}{ }^{h}$ at a point $A$ in a coordinate neighbourhood $U$. At the point $(A, p)$ in $\pi^{-1}(U)$, we can define a 1 -form $\sigma$ by 
Thus

$$
\sigma_{i}=p_{a} F_{i}^{a}, \quad \sigma_{\bar{i}}=0 .
$$

$$
\sigma=p_{a} F_{b}^{a} d x^{b}
$$

The exterior derivative of $\sigma$ is given by

$$
d \sigma=p_{a} \frac{\partial F_{b}{ }^{a}}{\partial x^{c}} d x^{c} \wedge d x^{b}+F_{b}^{a} d p_{a} \wedge d x^{b}
$$

so that if we write

$$
d \sigma=\frac{1}{2} \tau_{C B} d x^{C} \wedge d x^{B},
$$

where $\tau$ is skew-symmetric, (as before $x^{\bar{i}}$ means $p_{i}$ ) we have

$$
\begin{aligned}
\tau_{j i} & =p_{a}\left(\frac{\partial F_{i}{ }^{a}}{\partial x^{j}}-\frac{\partial F_{j}{ }^{a}}{\partial x^{i}}\right), \\
\tau_{\bar{j} i} & =F_{i}{ }^{j}, \\
\tau_{j \bar{i}} & =-F_{j}{ }^{i}, \\
\tau_{\bar{j} \bar{i}} & =0 .
\end{aligned}
$$

We write $F^{c}$ for the tensor field of type $(1,1)$ in ${ }^{c} T(M)$ whose components $\widetilde{F}_{B}{ }^{A}$ in $\pi^{-1}(U)$ are given by

Thus

$$
F_{B}^{A}=\tau_{B C} \varepsilon^{C A}
$$

$$
\begin{aligned}
& \tilde{F}_{i}{ }^{h}=F_{i}{ }^{h}, \quad \widetilde{F}_{i}^{h}=0 \\
& \widetilde{F}_{i}^{\bar{h}}=p_{a}\left(\frac{\partial F_{h}{ }^{a}}{\partial x^{i}}-\frac{\partial F_{i}{ }^{a}}{\partial x^{h}}\right), \widetilde{F}_{i}^{\bar{h}}=F_{h}{ }^{i} .
\end{aligned}
$$

We call $F^{c}$ the complete lift of the tensor field $F$. If $\omega \in \mathscr{T}_{1}^{0}(M)$, we have

$$
F^{C}\left(\omega^{v}\right)=(\omega F)^{v} \text {. }
$$

If $Z \in \mathscr{I}_{0}^{1}(M)$, we have

$$
F^{C}\left(Z^{C}\right)=(F Z)^{C}+\left(\mathcal{L}_{Z} F\right)^{V} .
$$

By Proposition 2, $F^{C}$ is completely determined by (14.3).

The action of $F^{C}$ on vertical vectors is completely determined by (14.2). If $G \in \mathscr{I}_{1}^{1}(M)$, then $G^{V}$ is a vertical vector in ${ }^{c} T(M)$ and

$$
F^{C}\left(G^{V}\right)=(G F)^{V} .
$$

If $\tilde{H} \in \mathscr{I}_{1}^{1}\left({ }^{c} T(M)\right)$ and

$$
\widetilde{H}\left(\omega^{V}\right)=(\omega F)^{V}
$$

for all $\omega \in \mathscr{I}_{1}^{0}(M)$ and some $F \in \mathscr{I}_{1}^{1}(M)$, we shall say that $\tilde{H}$ is projectable with projection $F$. In particular, $F^{C}$ is projectable with projection $F$.

Proposition 11. If $F \in \mathscr{I}_{1}^{1}(M)$ and $S \in \mathscr{I}_{2}^{1}(M)$, then 


$$
F^{C} S^{V}=(S F)^{V},
$$

where $S F \in \mathscr{I}_{2}^{1}(M)$ is defined by

$$
(S F)(X, Y)=S(X, F Y) .
$$

PRoof. If $Z \in \mathscr{I}_{0}^{1}(M)$, then, by (12.3) and (14.4),

But

$$
\begin{aligned}
\left(F^{c} S^{v}\right) Z^{c} & =F^{c}\left(S^{v} Z^{c}\right) \\
& =F^{c}\left(S_{Z}\right)^{v} \\
& =\left(S_{Z} F\right)^{v} .
\end{aligned}
$$

$$
(S F)^{V} Z^{C}=\left\{(S F)_{Z}\right\}^{V}
$$

and, since

$$
\left\{(S F)_{Z}\right\}(Y)=(S F)(Z, Y)=S(Z, F Y)=\left(S_{Z} F\right)(Y)
$$

for all $Y \in \mathscr{T}_{0}^{1}(M)$, it follows that

$$
\left\{(S F)_{z}\right\}^{V}=\left(S_{Z} F\right)^{V} .
$$

The required result now follows from Proposition 2.

Proposition 12. If $F \in \mathscr{I}_{1}^{1}(M)$ and $S \in \mathscr{I}_{2}^{1}(M)$, then

$$
S^{v} F^{C}=(S F)^{v}
$$

if and only if

$$
S(Z, F Y)=S(F Z, Y)
$$

for all $Z, Y \in \mathscr{I}_{0}^{1}(M)$.

Proof. Suppose that $Z \in I_{0}^{1}(M)$. Then, by (14.3), Proposition 10 and (12.3),

But, by (12.3),

$$
\begin{aligned}
\left(S^{v} F^{c}\right) Z^{c} & =S^{v}\left\{(F Z)^{C}+\left(\mathcal{L}_{Z} F\right)^{v}\right\} \\
& =S^{v}(F Z)^{c} \\
& =\left(S_{F Z}\right)^{v} .
\end{aligned}
$$

$$
(S F)^{V} Z^{C}=\left\{(S F)_{Z}\right\}^{V} .
$$

Now $S_{F Z}=(S F)_{Z}$ if and only if for all $Y \in \mathcal{I}_{0}^{1}(M)$ we have

$$
S_{F Z} Y=(S F)_{Z} Y:
$$

that is, if and only if

$$
S(F Z, Y)=S(Z, F Y) \text {. }
$$

Since $\left(S_{F Z}\right)^{V}=(S F)_{Z}^{V}$ if and only if $S_{F Z}=(S F)_{Z}$, the required result follows at once. 
$\S 15$. The complete lift of a skew-symmetric tensor field of type $(1,2)$.

Suppose now that $S$ is a skew-symmetric tensor of type $(1,2)$ in $M$ and that $S$ has components $S_{j i}^{h}$ at a point $A$ in a coordinate neighbourhood $U$. At the point $(A, p)$ in $\pi^{-1}(U)$, we can define a 2 -form $\sigma$ by

Thus

$$
\begin{array}{ll}
\sigma_{j i}=p_{a} S_{j i}{ }^{a}, & \sigma_{j i}=0, \\
\sigma_{j i}=0, & \sigma_{\overline{j i}}=0 .
\end{array}
$$

$$
\sigma=\frac{1}{2}-p_{a} S_{j i}{ }^{a} d x^{j} \wedge d x^{i}
$$

The exterior derivative $d \sigma$ of $\sigma$ is a 3 -form given by

$$
d \sigma=\frac{1}{2} p_{a} \frac{\partial S_{j i}{ }^{a}}{\partial x^{k}} d x^{k} \wedge d x^{j} \wedge d x^{i}+\frac{1}{2} S_{j i}{ }^{a} d p_{a} \wedge d x^{j} \wedge d x^{i} .
$$

Hence, if we write

$$
d \sigma=\frac{1}{6} \tau_{D C B} d x^{D} \wedge d x^{C} \wedge d x^{B}
$$

where $\tau$ is skew-symmetric in all pairs of suffixes and $x^{i}$ means $p_{i}$, we have

$$
\begin{aligned}
& \tau_{j i h}=p_{a}\left(\frac{\partial S_{i h}{ }^{a}}{\partial x^{j}}+\frac{\partial S_{h j}{ }^{a}}{\partial x^{i}}+\frac{\partial S_{j i}{ }^{a}}{\partial x^{h}}\right), \\
& \tau_{\overline{j i h}}=S_{i h^{j}}{ }^{h}, \tau_{j \overline{i n h}}=S_{h j}{ }^{i}, \quad \tau_{j i \bar{h}}=S_{j i}{ }^{h}, \\
& \tau_{\overline{j i} B}=0=\tau_{\bar{j} B \bar{h}}=\tau_{B i \bar{h}} .
\end{aligned}
$$

We write $S^{c}$ for the tensor field of type $(1,2)$ in ${ }^{c} T(M)$ whose components $\hat{S}_{C B}{ }^{A}$ in $\pi^{-1}(U)$ are given by

Thus

$$
\hat{S}_{C B}{ }^{A}=-\tau_{C B E} \varepsilon^{E A}
$$

$$
\begin{aligned}
& \hat{S}_{j i}{ }^{h}=S_{j i}{ }^{h}, \quad \hat{S}_{j \bar{i}}{ }^{h}=\hat{S}_{\bar{j} i}^{h}=\widetilde{S}_{\overline{j i}}{ }^{h}=0, \\
& \hat{S}_{j i}{ }^{\bar{h}}=-p_{a}\left(\frac{\partial S_{i h}{ }^{a}}{\partial x^{j}}+\frac{\partial S_{h j}{ }^{a}}{\partial x^{i}}+\frac{\partial S_{j i}{ }^{a}}{\partial x^{h}}\right), \\
& \widetilde{S}_{j i}^{\bar{h}}=S_{j h}{ }^{i}, \quad \widetilde{S}_{j i}^{\bar{h}}=S_{h i}{ }^{j}, \quad S_{j i}{ }^{\bar{h}}=0 .
\end{aligned}
$$

We call $S^{c}$ the complete lift of the tensor field $S$. If $\phi, \omega \in \mathscr{T}_{1}^{0}(M)$ and $Y, Z \in \mathcal{I}_{0}^{1}(M)$, we have

$$
\begin{gathered}
S^{c}\left(\phi^{V}, \omega^{V}\right)=0, \\
S^{c}\left(\omega^{V}, Z^{c}\right)=-\left(\omega S_{Z}\right)^{V}, \\
S^{C}\left(Y^{c}, Z^{c}\right)=(S(Y, Z))^{c}+\left\{\left(\mathcal{L}_{Z} S\right)_{Y}-\left(\mathcal{L}_{Y} S\right)_{Z}-S_{[Y, Z]}\right\}^{V}
\end{gathered}
$$

where $\left(\mathcal{L}_{Z} S\right)_{Y} \in \mathscr{I}_{1}^{1}(M)$ is given by 


$$
\left(\mathcal{L}_{Z} S\right)_{Y}(X)=\left(\mathcal{L}_{Z} S\right)(Y, X)
$$

and $S_{[Y, Z]} \in \mathscr{I}_{1}^{1}(M)$ is given by

$$
S_{[Y, Z]}(X)=S([Y, Z], X) \text {. }
$$

From Proposition 2 it follows that $S^{c}$ is completely determined by (15.3).

\section{$\S 16$. Theorems on structures in the cotangent bundle.}

We now apply our constructions of lifts of tensor fields to obtain theorems concerning the existence of certain types of structure in ${ }^{c} T(M)$. In our arguments, the torsion of two tensors of type $(1,1)$ plays an important part. If $F, G \in \mathscr{T}_{1}^{1}(M)$, the torsion $N_{F, G}$ of $F, G$ is the tensor field of type $(1,2)$ defined by

$$
\begin{aligned}
2 N_{F, G}(X, Y)= & {[F X, G Y]+[G X, F Y]+F G[X, Y]+G F[X, Y] } \\
& -F[X, G Y]-F[G X, Y]-G[X, F Y]-G[F X, Y]
\end{aligned}
$$

where $X, Y \in \mathscr{I}_{0}^{1}(M)$. (See [2], p. 37; we have introduced a factor $\frac{1}{2}$ for convenience.) It is easily seen that

$$
N_{F, G}=N_{G, F}
$$

and that $N_{F, G}$ is skew-symmetric. If we put $F=G$, we obtain the Nijenhuis tensor of $F$, given by

$$
N_{F, F}(X, Y)=[F X, F Y]+F^{2}[X, Y]-F[X, F Y]-F[F X, Y] \text {. }
$$

We shall abbreviate $N_{F, G}$ to $N$ whenever it is clear which tensor fields $F, G$ are involved.

If $F \in \mathscr{I}_{1}^{1}(M)$ and $F^{2}=-I$, where $I$ is the Kronecker tensor field (that is, the tensor field with components $\delta_{i}^{h}$ ), then $F$ is an almost complex structure on $M$. It is well-known that $F$ is integrable (that is, $F$ is obtainable from a complex structure on $M$ ) if and only if $N_{F, F}=0$.

If $F \in \mathscr{I}_{1}^{1}(M)$ and $F^{3}+F=0$, then $F$ is called an $f$-structure on $M$. (See [10], [11].)

Proposition 13. If $F$ is an almost complex structure on $M$ and $N=N_{F, F}$, then

$$
\begin{aligned}
N^{v} F^{C} & =(N F)^{V}, \\
(N F)^{V} F^{C} & =-N^{v} .
\end{aligned}
$$

Proof. By Proposition 12, it is sufficient to show that

and

$$
N(Z, F Y)=N(F Z, Y)
$$




$$
-N(Z, Y)=N(F Z, F Y)
$$

for all $Z, Y \in \mathscr{I}_{0}^{1}(M)$. This is a matter of direct verification, using $F^{2}=-I$.

Our next result establishes a connection between the complete lifts of two tensor fields $F, G \in \mathscr{I}_{1}^{1}(M)$ and the torsion of $F$ and $G$.

Proposition 14. If $F, G \in \mathscr{T}_{1}^{1}(M)$, then

where $N=N_{F, G}$.

$$
F^{C} G^{C}+G^{C} F^{C}=(F G+G F)^{C}+(2 N)^{V}
$$

Proof. Suppose that $X \in \mathscr{T}_{0}^{1}(M)$. By (14.3) and (14.4)

$$
\begin{aligned}
F^{c} G^{c} X^{c} & =F^{c}\left((G X)^{c}+\left(\mathcal{L}_{X} G\right)^{v}\right) \\
& =(F G X)^{c}+\left(\mathcal{L}_{G X} F\right)^{V}+\left\{\left(\mathcal{L}_{X} G\right) F\right\}^{v} \\
& =(F G)^{c} X^{C}-\left\{\mathcal{L}_{X}(F G)\right\}^{v}+\left(\mathcal{L}_{G X} F\right)^{V}+\left\{\left(\mathcal{L}_{X} G\right) F\right\}^{v} .
\end{aligned}
$$

Hence

$$
\left(F^{c} G^{C}+G^{C} F^{c}\right) X^{C}=(F G+G F)^{C} X^{C}+Q^{v}
$$

where $Q \in \mathscr{I}_{1}^{1}(M)$ is given by

$$
Q=\mathcal{L}_{G X} F+\left(\mathcal{L}_{X} G\right) F-\mathcal{L}_{X}(F G)+\mathcal{L}_{F X} G+\left(\mathcal{L}_{X} F\right) G-\mathcal{L}_{X}(G F) .
$$

By a well-known formula for Lie derivatives $([2]$, p. 32$)$ we have

$$
\begin{aligned}
Q Y= & {[G X, F Y]-F[G X, Y]+[X, G F Y]-G[X, F Y] } \\
& -[X, F G Y]+F G[X, Y]+[F X, G Y]-G[F X, Y] \\
& +[X, F G Y]-F[X, G Y]-[X, G F Y]+G F[X, Y]
\end{aligned}
$$

for any $Y \in \mathscr{I}_{0}^{1}(M)$, from which it follows that

By (12.3),

$$
Q Y=2 N(X, Y) \text {. }
$$

But

$$
N^{V} X^{C}=\left(N_{X}\right)^{V}
$$

\section{But}

so that

$$
2 N_{X}(Y)=2 N(X, Y)=Q Y
$$

Hence

$$
2 N_{X}=Q
$$

$$
Q^{v}=2 N^{v} X^{c}
$$

so that, by (16.4), the actions of $F^{c} G^{C}+G^{C} F^{C}$ and $(F G+G F)^{C}+2 N^{v}$ on $X^{c}$ are the same.

The required result now follows from Proposition 2.

Proposition 15. If $F \in \mathscr{I}_{1}^{1}(M)$, then

$$
\left(F^{C}\right)^{2}=\left(F^{2}\right)^{C}+\left(N_{F, F}\right)^{V} .
$$


This is an immediate corollary of Proposition 14.

Proposition 16. If $F \in \mathscr{I}_{1}^{1}(M)$, then

$$
\left(F^{C}\right)^{3}=\left(F^{3}\right)^{C}+(2 T-F N)^{V}
$$

where $T$ is the torsion of $F$ and $F^{2}$, and $N$ is the Nijenhuis tensor of $F$.

Proof. By Propositions 15 and 11,

By (16.3),

$$
\begin{aligned}
\left(F^{C}\right)^{3} & =F^{C}\left(F^{2}\right)^{C}+F^{C} N^{V} \\
& =F^{C}\left(F^{2}\right)^{C}+(N F)^{V} .
\end{aligned}
$$

$$
F^{C}\left(F^{2}\right)^{C} X^{C}=\left(F^{3}\right)^{C} X^{C}+\left\{\left(\mathcal{L}_{X} F^{2}\right) F+\left(\mathcal{L}_{F^{2} X} F\right)-\mathcal{L}_{X} F^{3}\right\}^{V}
$$

so that, using (16.7) and (12.3)

where

$$
\left(F^{C}\right)^{3} X^{C}=\left(F^{3}\right)^{C} X^{C}+R^{V}
$$

We have

$$
R=\left(\mathcal{L}_{X} F^{2}\right) F+\mathcal{L}_{F^{2}{ }_{X}} F-\mathcal{L}_{X} F^{3}+(N F)_{X} .
$$

$$
\begin{aligned}
R Y= & {\left[X, F^{3} Y\right]-F^{2}[X, F Y]+\left[F^{2} X, F Y\right]-F\left[F^{2} X, Y\right] } \\
& -\left[X, F^{3} Y\right]+F^{3}[X, Y]+\left[F X, F^{2} Y\right]+F^{2}[X, F Y] \\
& -F\left[X, F^{2} Y\right]-F[F X, F Y] \\
= & {\left[F X, F^{2} Y\right]+\left[F^{2} X, F Y\right]+2 F^{3}[X, Y] } \\
& -F\left[X, F^{2} Y\right]-F\left[F^{2} X, Y\right]-F^{2}[X, F Y]-F^{2}[F X, Y] \\
& -F[F X, F Y]-F^{3}[X, Y]+F^{2}[X, F Y]+F^{2}[F X, Y] \\
= & 2 T(X, Y)-F N(X, Y) \\
= & 2 T_{X}(Y)-(F N)_{X}(Y) \quad \text { for any } Y \in \mathscr{I}_{0}^{1}(M) .
\end{aligned}
$$

Hence, by (16.8) and (12.3)

$$
\begin{aligned}
\left(F^{C}\right)^{3} X^{C} & =\left(F^{3}\right)^{C} X^{C}+\left(2 T_{X}-(F N)_{X}\right)^{V} \\
& =\left(F^{3}\right)^{C} X^{C}+(2 T-F N)^{V} X^{c} .
\end{aligned}
$$

This proves Proposition 16.

Proposition 17. If $F, G \in \mathscr{I}_{1}^{1}(M)$ and $\tilde{N}$ is the torsion of $F^{C}$ and $G^{C}$, then

$$
\tilde{N}=N^{c}
$$

where $N$ is the torsion of $F$ and $G$.

This result can be proved (using Proposition 2) by means of a straightforward but somewhat lengthy computation.

We come now to our main theorems. 
THEOREM 1. Let $F$ be an almost complex structure on $M$. Then the complete lift $F^{c}$ is an almost complex structure on ${ }^{c} T(M)$ if and only if $F$ is integrable.

Proof. Since $F$ is an almost complex structure, we have $F^{2}=-I$. Hence, by Proposition 15,

$$
\left(F^{C}\right)^{2}=(-I)^{C}+N^{V}
$$

where $N$ is the Nijenhuis tensor of $F$. Since the complete lift of $I$ in $M$ is the Kronecker tensor field $\widetilde{I}$ in ${ }^{c} T(M)$, we have $\left(F^{c}\right)^{2}=-\widetilde{I}$ if and only if $N^{v}=0$. Since $N^{v}=0$ is equivalent to $N=0$ it follows that $F^{C}$ is an almost complex structure in ${ }^{c} T(M)$ if and only if $N=0$.

THEOREM 2. If $F$ is an integrable almost complex structure on $M$, then the complete lift $F^{c}$ is an integrable almost complex structure on ${ }^{c} T(M)$.

Proof. By Theorem $1, F^{C}$ is an almost complex structure. Since $F$ is integrable, the Nijenhuis tensor of $F$ is zero. Hence, by Proposition 17, the Nijenhuis tensor of $F^{c}$ is also zero.

THEOREM 3. Let $F$ be an almost complex structure on $M$, with $N$ the Nijenhuis tensor of $F$. Then

$$
F^{C}+\frac{1}{2}(N F)^{V}
$$

is an almost complex structure on ${ }^{c} T(M)$.

This theorem is due to Satô [8].

Proof. Using Proposition 10, we have

$$
\begin{aligned}
\left\{F^{C}+\frac{1}{2}(N F)^{V}\right\}^{2} & =\left(F^{C}\right)^{2}+\frac{1}{2} F^{C}(N F)^{V}+\frac{1}{2}(N F)^{V} F^{C} \\
& =\left(F^{C}\right)^{2}+\frac{1}{2}\left(N F^{2}\right)^{V}+\frac{1}{2}\left(N F^{2}\right)^{V}
\end{aligned}
$$

by Propositions 11 and 13. Since $F^{2}=-I$, we get, using Proposition 15,

$$
\left\{F^{C}+\frac{1}{2}(N F)^{V}\right\}^{2}=\left(F^{C}\right)^{2}-N^{V}=\left(F^{2}\right)^{C}=-\tilde{I} .
$$

THEOREM 4. The almost complex structure $F^{c}+\frac{1}{2}(N F)^{V}$ on ${ }^{c} T(M)$ (see Theorem 3) is integrable if and only if $F$ is integrable.

Proof. If $F$ is integrable, then $N=0$ and so $F^{c}+\frac{1}{2}(N F)^{V}=F^{c}$; by Theorem $2, F^{C}$ is also integrable.

Suppose conversely that $F^{C}+\frac{1}{2}(N F)^{V}$ is integrable. Then the Nijenhuis tensor of $F^{C}+\frac{1}{2}(N F)^{V}$ in ${ }^{c} T(M)$ is zero. By a direct if somewhat lengthy computation (which makes use of the propositions proved in $\S 13$ ) we can 
show that the Nijenhuis tensor $\tilde{N}$ of $F^{C}+\frac{1}{2}(N F)^{V}$ satisfies

$$
\tilde{N}\left(X^{C}, Y^{c}\right)=\{N(X, Y)\}^{C}+P^{v}
$$

where $P$ is the tensor field of type $(1,1)$ in $M$ given by

$$
\begin{aligned}
2 P(Z)= & N(Y,[X, Z])-N(X,[Y, Z])+N(X, F[F Y, Z]) \\
& -N(Y, F[F X, Z])+N([F Y, X], F Z)-N([F X, Y], F Z) \\
& +[Y, N(X, Z)]-[X, N(Y, Z)]+F[F Y, N(X, Z)] \\
& -F[F X, N(Y, Z)]+\frac{1}{2} N(Y, N(X, Z))-\frac{1}{2} N(X, N(Y, Z)) .
\end{aligned}
$$

Since $\tilde{N}$ is zero, we get

$$
\{N(X, Y)\}^{C}+P^{v}=0 .
$$

But this shows that the vector $\{N(X, Y)\}^{C}$ is vertical; since the complete lift of a non-zero vector cannot be vertical, it follows that $N(X, Y)=0$. This holds for all $X, Y \in \mathscr{I}_{0}^{1}(M)$ and so $N=0$. Hence $F$ is integrable.

It is of some interest to note that the expression for $2 P(Z)$ is not linear in $X$ and $Y$. If we write $Q(X, Y, Z)$ for $P(Z)$, we find that

$$
Q(f X, g Y, Z)=f g Q(X, Y, Z)+(f(Z g)+g(Z f)) N(X, Y) .
$$

Theorem 5. Let $F$ be an $f$-structure on $M$. Let $N$ be the Nijenhuis tensor of $F$ and let $T$ be the torsion of $F$ and $F^{2}$. Then $F^{C}$ is an $f$-structure on $M$ if and only if

or, equivalently,

$$
2 T=F N,
$$

$$
N(X, F Y)+N(F X, Y)+F N(X, Y)=0
$$

for all $X, Y \in I_{0}^{1}(M)$.

ProOF. Since $F^{3}+F=0$, it follows from Proposition 16 that

$$
\left(F^{C}\right)^{3}+F^{C}=\left(F^{3}\right)^{C}+F^{C}+(2 T-F N)^{V}=(2 T-F N)^{V} .
$$

Hence $F^{C}$ is an $f$-structure if and only if $(2 T-F N)^{V}=0$, which is equivalent to $2 T=F N$.

To prove the last part, we simply verify that

$$
N(X, F Y)+N(F X, Y)+F N(X, Y)=(2 T-F N)(X, Y)
$$

for all $X$ and $Y$.

THEOREM 6. Let $F$ be an $f$-structure on $M$, let $N$ be the Nijenhuis tensor of $F$ and let $T$ be the torsion of $F$ and $F^{2}$. Then

$$
F^{C}+\left\{(F N-2 T)\left(I+\frac{3}{2} F^{2}\right)\right\}^{v}
$$


is an $f$-structure on ${ }^{c} T(M)$.

Proof. Write

$$
P=(F N-2 T)\left(I+\frac{3}{2} F^{2}\right) .
$$

If $X \in \mathscr{I}_{0}^{1}(M)$, then

$$
\left(F^{c}+P^{v}\right) X^{c}=(F X)^{c}+\left(\mathcal{L}_{X} F\right)^{V}+\left(P_{X}\right)^{v}
$$

by (12.3) and (14.3). Hence, by Proposition 10

and similarly

$$
\begin{aligned}
\left(F^{C}+P^{v}\right)^{2} X^{c} & =\left(F^{c}\right)^{2} X^{c}+F^{c} P_{X}^{v}+P^{v}(F X)^{C} \\
& =\left(F^{C}\right)^{2} X^{C}+\left(P_{X} F\right)^{v}+\left(P_{F X}\right)^{v}
\end{aligned}
$$

$$
\left(F^{C}+P^{V}\right)^{3} X^{C}=\left(F^{C}\right)^{3} X^{C}+\left(P_{X} F^{2}\right)^{V}+\left(P_{F X} F\right)^{V}+\left(P_{F^{2} X}\right)^{V} .
$$

Hence, by Proposition 16,

$$
\left(F^{C}+P^{V}\right)^{3} X^{C}=\left(F^{3}\right)^{C} X^{C}+(2 T-F N)_{X}{ }^{V}+\left(P_{X} F^{2}\right)^{V}+\left(P_{F X} F\right)^{V}+\left(P_{F^{2} X}\right)^{V} .
$$

Since $F^{3}=-F$, it follows that

$$
\left(F^{C}+P^{v}\right)^{3} X^{C}=-\left(F^{C}+P^{v}\right) X^{C}
$$

for all $X$ if and only if

$$
P_{X}+P_{X} F^{2}+P_{F X} F+P_{F^{2} X}=(F N-2 T)_{X}
$$

for all $X$. This condition is equivalent to

$$
\begin{array}{r}
P(X, Y)+P\left(X, F^{2} Y\right)+P(F X, F Y)+P\left(F^{2} X, Y\right) \\
=F N(X, Y)-2 T(X, Y)
\end{array}
$$

for all $X, Y \in \mathscr{I}_{0}^{1}(M)$. With $P$ defined by (16.10), a straightforward verification can be used to prove that $(16.11)$ is satisfied. Hence $\left(F^{c}+P^{v}\right)^{3} X^{c}+\left(F^{c}+P^{v}\right) X^{c}$ $=0$, so that (using Proposition 2 once more) we have

$$
\left(F^{C}+P^{v}\right)^{3}+\left(F^{c}+P^{v}\right)=0 .
$$

$\S 17$. The Riemann extension and the complete lift of a symmetric affine connection in $M$.

Let $\nabla$ be a symmetric affine connection in $M$. Let $A$ be a point of $M$ and let $U, U^{*}$ be coordinate neighbourhoods containing $A$. We write $\Gamma_{j i}^{h}$ and $\Gamma_{j i}^{* h}$ for the components of $\nabla$ relative to $U$ and $U^{*}$ respectively. Then the tensor field of type $(0,2)$ in ${ }^{c} T(M)$ whose components $\tilde{g}_{C B}$ in $\pi^{-1}(U)$ are given by

$$
\begin{aligned}
& \tilde{g}_{j i}=-2 p_{a} \Gamma_{j i}^{a}, \\
& \tilde{g}_{\overline{j i}}=\delta_{i}^{j}=\tilde{g}_{i \bar{j}}, \\
& \tilde{g}_{j \bar{i}}=0
\end{aligned}
$$


has components $\tilde{g}_{C B}^{*}$ in $\pi^{-1}\left(U^{*}\right)$ given by

$$
\begin{aligned}
& \tilde{g}_{j i}^{*}=-2 p_{a}^{*} \Gamma_{j i}^{* a}, \\
& \tilde{g}_{\overline{j i}}^{*}=\delta_{i}^{j}=\tilde{g}_{i \bar{j}}^{*}, \\
& \tilde{g}_{j i}^{*}=0 .
\end{aligned}
$$

We call this tensor field the Riemann extension of the connection $\nabla$ and denote it by $\nabla^{R}$ (see [4], [5], [6]). We have

$$
\begin{aligned}
& \nabla^{R}\left(\phi^{V}, \omega^{V}\right)=0 \\
& \nabla^{R}\left(X^{C}, \omega^{V}\right)=(\omega(X))^{V} \\
& \nabla^{R}\left(X^{c}, Y^{c}\right)=-\left(\nabla_{X} Y+\nabla_{Y} X\right)^{V} .
\end{aligned}
$$

By Proposition 2, the tensor field $\nabla^{R}$ is completely determined by the last of these three conditions.

Let $\nabla^{C}$ be the Levi-Civita connection determined by $\nabla^{R}$. We call $\nabla^{C}$ the complete lift of $\nabla$. The components $\tilde{\Gamma}_{C B}^{A}$ of $\nabla^{C}$ in $\pi^{-1}(U)$ are given by

$$
\begin{aligned}
& \tilde{\Gamma}_{j i}^{h}=\Gamma_{j i}^{h}, \quad \tilde{\Gamma}_{j \bar{i}}^{h}=0=\tilde{\Gamma}_{\overline{j i}}^{h}=\tilde{\Gamma}_{\overline{j i}}^{h}, \\
& \tilde{\Gamma}_{j i}^{\bar{h}}=p_{a}\left(\partial_{h} \Gamma_{j i}^{a}-\partial_{j} \Gamma_{i h}^{a}-\partial_{i} \Gamma_{j h}^{a}+2 \Gamma_{h b}^{a} \Gamma_{j i}^{b}\right), \\
& \tilde{\Gamma}_{j \bar{i}}^{\bar{h}}=-\Gamma_{j h}^{i}, \quad \tilde{\Gamma}_{\bar{j} i}^{\bar{h}}=-\Gamma_{h i}^{j}, \quad \tilde{\Gamma}_{\overline{j i}}^{\bar{h}}=0 .
\end{aligned}
$$

Proposition 18. Covariant differentiation with respect to the connection $\nabla^{C}$ in ${ }^{c} T(M)$ satisfies the following properties:

$$
\begin{aligned}
& \nabla_{\psi}^{C} v \omega^{V}=0, \quad \nabla_{X}^{C} c \omega^{V}=\left(\nabla_{X} \omega\right)^{V}, \\
& \nabla_{\psi}^{C} v F^{v}=(\psi F)^{V}, \quad \nabla_{X}^{C} F^{v}=\left(\nabla_{X} F-(\nabla X) F\right)^{V}, \\
& \nabla_{\psi}^{C} Y^{c}=-(\psi(\nabla Y))^{V}, \\
& \nabla_{X}^{C} Y^{c}=\left(\nabla_{X} Y\right)^{C}+\left\{(\nabla X)(\nabla Y)+(\nabla Y)(\nabla X)-K_{X} Y-K_{Y} X\right\}^{V}
\end{aligned}
$$

where $\psi, \omega \in \mathscr{I}_{1}^{0}(M), X, Y \in \mathscr{I}_{0}^{1}(M), F \in \mathscr{I}_{1}^{1}(M), K$ is the curvature tensor of $\nabla$ and $K_{X} \in \mathscr{I}_{2}^{1}(M)$ is given by

$$
\left(K_{X} Y\right)(Z)=K(X, Z) Y .
$$

Proof. These formulae can be obtained directly from formulae (17.3).

An alternative expression for $\nabla_{X}^{C} Y^{C}$ is

$$
\left(\nabla_{X} Y\right)^{C}+\left\{\nabla\left(\nabla_{X} Y+\nabla_{Y} X\right)-\left(\nabla_{X} \nabla Y+\nabla_{Y} \nabla X\right)\right\}^{V} .
$$

This can be proved from Proposition 18 by using the identity

$$
\nabla \nabla_{X} Y-\nabla_{X} \nabla Y=(\nabla Y)(\nabla X)-K_{X} Y \text {. }
$$

Proposition 19. Let $K$ be the curvature tensor of $\nabla^{c}$. Then if $\phi, \phi, \omega$ 
$\in \mathscr{I}_{1}^{0}(M)$ and $X, Y, Z \in \mathscr{I}_{0}^{1}(M)$, we have

$$
\begin{aligned}
& K\left(\phi^{V}, \phi^{v}\right) \omega^{V}=0, \quad K\left(\phi^{V}, \phi^{V}\right) Z^{c}=0, \\
& K\left(X^{c}, \phi^{v}\right) \omega^{V}=0, \quad K\left(X^{c}, \phi^{v}\right) Z^{c}=+\left(\phi K_{Z} X\right)^{v} \\
& K\left(X^{c}, Y^{c}\right) \omega^{V}=-(\omega(K(X, Y)))^{V} \\
& K\left(X^{c}, Y^{c}\right) Z^{c}=(K(X, Y) Z)^{c} \\
& \quad+\left\{\nabla(K(X, Y) Z)-(\nabla K)_{(X, Z)} Y+(\nabla K)_{(Y, Z)} X+(\nabla Z) K(X, Y)\right\}^{v}
\end{aligned}
$$

where

$$
(\nabla K)_{(X, Z)}(U)=(\nabla K)(U, X, Z)
$$

These formulae follow from the formulae for $\nabla^{c}$ given in Proposition 18,

Tokyo Institute of Technology

and

University of Aberdeen, Scotland

\section{Bibliography}

[1] P. Dombrowski, On the geometry of tangent bundles, J. reine angew. Math., 210 (1962), 73-88.

[2] S. Kobayashi and K. Nomizu, Foundations of differential geometry, Interscience Tract, No. 15, 1963.

[3] A. J. Ledger and K. Yano, The tangent bundle of a locally symmetric space, J. London Math. Soc., 40 (1965), 487-492.

[4] E. M. Patterson, Simply harmonic Riemann extensions, J. London Math. Soc., 27 (1952), 102-107.

[5] E. M. Patterson, Riemann extensions which have Kähler metrics, Proc. Roy. Soc. Edinburgh Sect. A, 64 (1954), 113-126.

[6] E. M. Patterson and A. G. Walker, Riemann extensions, Quart. J. Math. Oxford Ser., 3 (1952), 19-28.

[7] S. Sasaki, On the differential geometry of tangent bundles of Riemannian manifolds, Tôhoku Math. J., 10 (1958), 338-354.

[8] I. Satô, Almost analytic vector fields in almost complex manifolds, Tôhoku Math. J., 17 (1965), 185-199.

[9] P. Tondeur, Structure presque kählérienne naturelle sur le fibre des vecteurs covariants d'une variété riemannienne, C. R. Acad. Sci. Paris, 254 (1962), 407-408.

[10] K. Yano, On a structure $f$ satisfying $f^{3}+f=0$, Technical Reports, No. 2 (1961), University of Washington.

[11] K. Yano, On a structure defined by a tensor field $f$ of type $(1,1)$ satisfying $f^{3}+f=0$, Tensor, N.S., 14 (1963), 9-19.

[12] K. Yano and E. T. Davies, On tangent bundles of Finsler and Riemannian manifolds, Rend. Circ. Mat. Palermo, 12 (1963), 211-228. 
[13] K. Yano and S. Ishihara, Horizontal lifts of tensor fields and connections to tangent bundles, to appear in J. Math. Mech..

[14] K. Yano and S. Kobayashi, Prolongations of tensor fields and connections to tangent bundles, I. General theory, J. Math. Soc. Japan, 18 (1966), 194-210. II. Affine automorphisms, ibid. 18 (1966), 236-246.

[15] K. Yano and A.J. Ledger, Linear connections on tangent bundles, J. London Math. Soc., 39 (1964), 495-500. 